\title{
A simple unified view of branching process statistics: random walks in balanced logarithmic potentials
}

\author{
Serena di Santo, ${ }^{1,2,3}$ Pablo Villegas, ${ }^{1}$ Raffaella Burioni, ${ }^{2,3}$ and Miguel A. Muñoz ${ }^{1}$ \\ ${ }^{1}$ Departamento de Electromagnetismo y Física de la Materia e Instituto Carlos I de \\ Física Teórica y Computacional. Universidad de Granada. E-18071, Granada, Spain \\ ${ }^{2}$ Dipartimento di Fisica e Scienza della Terra, Università di Parma, via G.P. Usberti, 7/A - 43124, Parma, Italy \\ ${ }^{3}$ INFN, Gruppo Collegato di Parma, via G.P. Usberti, 7/A - 43124, Parma, Italy
}

\begin{abstract}
We revisit the problem of deriving the mean-field values of avalanche exponents in systems with absorbing states. These are well-known to coincide with those of un-biased branching processes. Here, we show that for at least 4 different universality classes (directed percolation, dynamical percolation, the voter model or compact directed percolation class, and the Manna class of stochastic sandpiles) this common result can be obtained by mapping the corresponding Langevin equations describing each of them into a random walker confined to the origin by a logarithmic potential. We report on the emergence of non-universal continuously-varying exponent values stemming from the presence of small external driving -that might induce avalanche merging- that, to the best of our knowledge, has not been noticed in the past. Many of the other results derived here appear in the literature as independently derived for individual universality classes or for the branching process itself. Still, we believe that a simple and unified perspective as the one presented here can help (i) clarify the overall picture, (ii) underline the super-universality of the behavior as well as the dependence on external driving, and (iii) avoid the common existing confusion between unbiased branching processes (equivalent to a random walker in a balanced logarithmic potential) and standard (un-confined) random walkers.
\end{abstract}

Directed percolation (DP) is the paradigmatic example of a very large class of systems -including catalytic reactions, growing interfaces in random media, damage spreading, epidemic dynamics, and turbulence, to name but a few- exhibiting a phase transition separating a quiescent or absorbing state from an active one [1 6 . The essence of this very robust universality class - which, curiously enough, had to wait long for experimental backing [7- is parsimoniously encoded in the following Langevin equation [4, 6, 8, 9]

$\dot{\rho}(\mathbf{r}, t)=a \rho(\mathbf{r}, t)-b \rho^{2}(\mathbf{r}, t)+D \nabla^{2} \rho(\mathbf{r}, t)+\sqrt{\rho(\mathbf{r}, t)} \eta(\mathbf{r}, t)$,

where $\rho(\mathbf{r}, t)$ is the density of activity at coordinates $\mathbf{r}$ and time $t, a$ is the control parameter regulating the distance to the critical point, $b$ and $D$ are constants, and $\eta(t)$ is a Gaussian white noise of variance $\sigma^{2}$. Critical exponents, scaling functions, and, in general, all critical features can be obtained using Eq.(1) as a starting point. The most preponderant aspect of this equation, distinguishing it from other classes, as for instance the Ising class [10], is the $\sqrt{\rho}$ factor in the noise amplitude. This square-root noise term stems from the "demographic" nature of the particle-number fluctuations; and it imposes that there are no fluctuations in the absence of activity, as corresponds to the absorbing state [11].

The same type of demographic noise also appears in other slightly different universality classes, such as (i) the voter-model or neutral class describing the dynamics of neutral theories in which two symmetric competing states are possible [1, 12, 14; in this class there is no deterministic force except for diffusion, and the noise amplitude is different from zero only at the interfaces separating the two absorbing states e.g. at $\rho=0$ and $\rho=1$, i.e. $\quad \dot{\rho}(\mathbf{r}, t)=D \nabla^{2} \rho(\mathbf{r}, t)+\sqrt{\rho(\mathbf{r}, t)(1-\rho(\mathbf{r}, t))}$ [14]; (ii) the dynamical percolation class [15, 16] -in which re-activation of sites cannot occur and, as a consequence, the non-linear term in Eq.(1) needs to be replaced by a non-Markovian term $-\rho(\mathbf{r}, t) \int_{-\infty}^{t} d t^{\prime} \rho\left(\mathbf{r}, t^{\prime}\right)$ keeping track of past activity while the noise term remains unchanged, and (iii) the Manna class of systems with many absorbing states such as sandpiles in which an additional conservation law -that can be encapsulated in an additional term $-\rho(\mathbf{r}, t) \int_{-\infty}^{t} d t \nabla^{2} \rho(\mathbf{r}, t)$ [17, 18]- exists, while the noise term remains as in directed percolation.

All systems with absorbing states, including these four classes and some other more infrequent ones, not specified here- share the common feature of exhibiting avalanching behavior, meaning that if the absorbing state is perturbed by a localized seed of activity, this can trigger a cascade of events before falling back again into the absorbing state. It is common knowledge that avalanches turn out to be scale invariant at critical points; in particular, the avalanche-size $(S)$ and avalanche-duration $(T)$ probability distribution functions can be written at criticality as

$$
\begin{aligned}
P(S) & \sim S^{-\tau} \mathcal{G}_{\mathcal{S}}\left(S / S_{C}\right) \\
F(T) & \sim T^{-\alpha} \mathcal{G}_{\mathcal{T}}\left(T / T_{C}\right),
\end{aligned}
$$

where $\mathcal{G}_{\mathcal{S}}\left(S / S_{C}\right)$ and $\mathcal{G}_{\mathcal{T}}\left(T / T_{C}\right)$ are cut-off functions, and the cut-off scales, $S_{C}$ and $T_{C}$, depend only on system size right at the critical point, and on the distance to criticality away from it [19]. Similarly, the averaged avalanche size scales with the duration as $\langle S\rangle \sim T^{\gamma}$, where the ex- 
ponent $\gamma$ needs to obey the scaling relation [20, 21],

$$
\gamma=\frac{\alpha-1}{\tau-1} .
$$

In particular, for avalanches propagating in high dimensional systems (or in densely connected networks) meanfield exponent values $\tau=3 / 2, \alpha=2$ and $\gamma=2$ are obtained for all systems with absorbing states. A compilation of avalanche exponents for different dimensions and universality classes, as well as scaling relationships, can be found in 22 25].

In order to explicitly compute these exponent values, textbooks usually resort to the (Galton-Watson) branching process [1, 2, 26, 27]. In this, each node of a tree has two branches emerging out of it; from an occupied/active node at time/generation $n$ each of its two out-branches (at time/generation $n+1$ ) are occupied/active with probability $p$ or left empty with complementary $(1-p)$. Observe that this is just a variant of directed percolation running on a regular tree (see Figure 1). For illustration and completeness, we now present a very simple derivation of its associated avalanche distribution functions.

To compute $P(S)$-where $S$ is the total number of occupied/active nodes before the process comes to its endone just needs to evaluate the total number of connected trees of size $S$, which is nothing but the Catalan number 28,

$$
C(S)=\frac{1}{S}\left(\begin{array}{c}
2 S \\
S-1
\end{array}\right)
$$

and multiply it for the probability of each one to occur, $p^{S-1}(1-p)^{S+1}$. Evaluating the resulting expression $P(S, p)=(2 S) ! /((S+1) ! S !) p^{S-1}(1-p)^{S+1}$ in the Stirling approximation for $S>>1$, one readily obtains

$$
P(S, p)=\frac{\mathcal{N}}{\sqrt{\pi}} S^{-3 / 2}(4 p(1-p))^{S},
$$

where $\mathcal{N}$ is a normalization constant; in particular, this becomes a power law at the critical point $p=1 / 2$ : $P(S, 1 / 2)=\frac{\mathcal{N}}{\sqrt{\pi}} S^{-3 / 2}$, implying $\tau=3 / 2$. The exponent $\gamma$ can also be derived using the statistics of branch lengths in Catalan trees of a given size [29], leading readily to the result $\gamma=2$; and from this, using the scaling relation Eq.(3), one obtains $\alpha=2$.

These results for the branching-process avalanche statistics can be derived in a more systematic way for different types of underlying regular or random tree topologies- within the generating function formalism 30 32]; indeed, already back in 1949 Otter computed the solution for the case of a Poissonian distribution of branches per node [33. Given that the result, e.g. a power-law with exponent $3 / 2$ for the size distribution, is much more general than any specific branching process in any specific tree-like topology, it is appealing from a theoretical point of view to derive an even more general proof of these results, covering all cases at once. From a slightly different perspective, relying on field theory and scaling
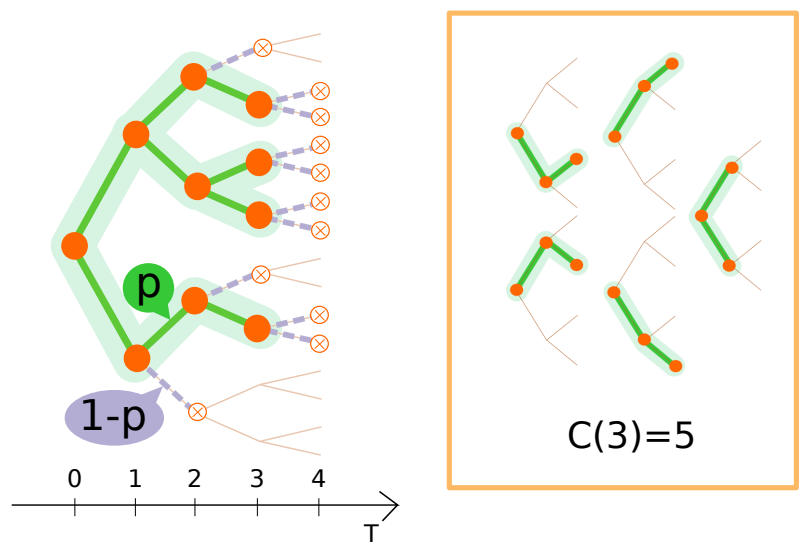

FIG. 1. Left: Illustration of a realization of the un-biased branching process, showing (highlighted) an avalanche of size $S=10$ and duration $T=3$, together with the structure of the underlying rooted binary tree on top of which it unfolds. Right: Visualization of the 5 possible paths of $S=3$ as counted by the Catalan number $C(3)=5$.

arguments [22, 34, 35] the whole set of exponent values can be obtained for each specific universality class, but again, the result -being common to all classes, i.e. super-universal- should be amenable for a more generic explanation.

The common feature shared by all the Langevin equations of the different classes of systems with absorbing states, as already mentioned above, is the presence of a demographic, square-root, noise amplitude. As a matter of fact -as illustrated in more detail in Appendix A- in the mean-field limit it is easy to derive a common and unique effective Langevin equation for all classes of systems with absorbing states at criticality, as

$$
\dot{\rho}=\sqrt{\rho} \xi(t),
$$

where $\rho$ is the overall activity and $\xi(t)$ is a Gaussian white noise with zero mean and $\left\langle\xi(t) \xi\left(t^{\prime}\right)\right\rangle=2 \sigma^{2} \delta\left(t-t^{\prime}\right)$ which needs to be interpreted in the Itô sense in order to guarantee that $\rho=0$ is an absorbing state [36, 37]. We refer to Eq. (6) as "demographic random walker" (DRW). To avoid the complications of the Itô calculus, we write the equivalent equation in the Stratonovich interpretation [36, 37]:

$$
\dot{\rho}=-\frac{\sigma^{2}}{2}+\sqrt{\rho} \eta(t)
$$

where now $\left\langle\eta(t) \eta\left(t^{\prime}\right)\right\rangle=\frac{\sigma^{2}}{2} \delta\left(t-t^{\prime}\right)$. Using now standard calculus to change variables to $x=\sqrt{\rho}$ directly gives [38]

$$
\dot{x}=-\frac{\sigma^{2}}{4 x}+\eta(t) .
$$

The resulting equation is just a particular case of a onedimensional random walker (RW) moving in a logarith- 

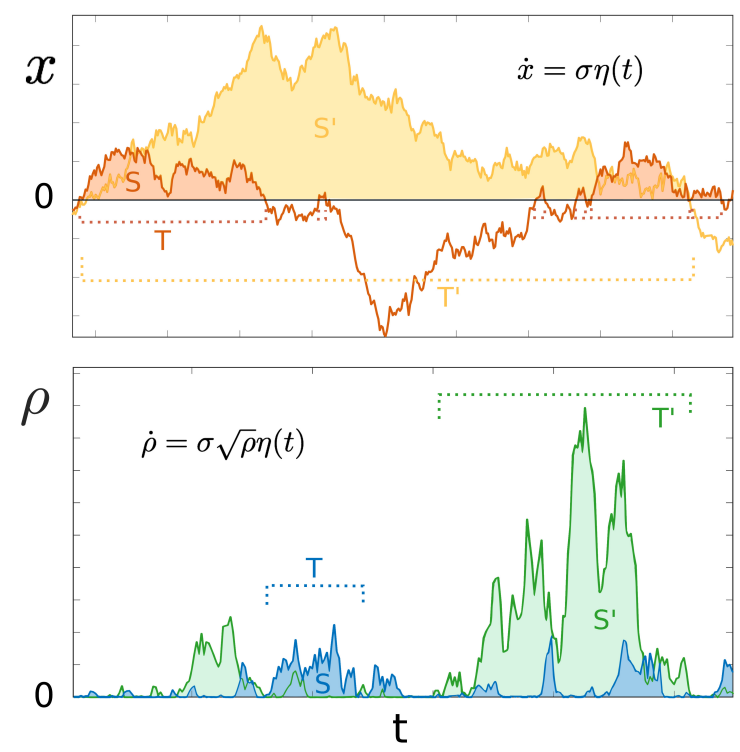

FIG. 2. Illustration of the time evolution of a standard random walk (RW) and a demographic random walk (DRW); each color corresponds to a different realization. Upper panel: standard RW that, in principle, can freely cross the origin. Avalanches start and end when the walker crosses the origin. Lower panel: the DRW can be represented as a stochastic RW moving in a balanced logarithmic potential that keeps the walker bounded to the origin. Since the variable is always strictly positive, the avalanches can be defined as the activity over a threshold $\epsilon \rightarrow 0$.

mic potential $U(x)=\lambda \log x$, i.e.

$$
\frac{d x}{d t}=-\frac{d U(x)}{d x}+\eta(t)=-\frac{\lambda}{x}+\eta(t)
$$

where $\lambda$ is a positive constant and, in general, $\left\langle\eta(t) \eta\left(t^{\prime}\right)\right\rangle=2 \mu \delta\left(t-t^{\prime}\right)$, with $\mu$ a generic positive constant. Observe tha $\mathrm{Eq}(8)$ corresponds to the particular case, $\lambda=\mu=\sigma^{2} / 4$-that we call balanced- in which the ratio between the amplitudes of the logarithmic potential and the noise-correlation amplitude, $\mu$, is equal to unity: $\beta \equiv \lambda / \mu=1$. This perfect balance between the deterministic-force and stochastic coefficients is essential for what follows, as we shall see. More in general, let us remark that, in the presence of an external field -allowing for the spontaneous generation of activity at a fixed rate $h$ - Eq.(7) needs to be complemented with an additional $+h$ term. Upon changing variables, this implies $\beta=1 \rightarrow 1-h / \mu$, in Eq. (9) and thus, in the presence of external driving, the perfect balance between coefficients breaks down.

To compute avalanche exponents from Eq. (9), let us define an avalanche as a random walk $x(T)$, starting at $x(t=0)=0^{+}$and returning for the first time to the origin at time $T, x(T)=0$ (see Figure 2). The distribution or its return times is nothing but $F(T)$ as defined in Eq.22). The problem of computing such a return-time distribution for the random walk in a logarithmic potential, i.e. by Eq. (8), was solved by A. Bray [39] and revisited by $\mathrm{F}$. Colaiori in the context of Barkhaussen crackling noise [40. The solution requires writing down the equivalent Fokker Planck equation for the Langevin dynamics, with a delta-like initial condition centered at a value slightly larger than $x=0$, and computing the probability flux $F$ at the origin as a function of the time $T$ (more detailed sketch of the analysis is presented in Appendix B for the sake of completeness). The resulting first-return probability distribution function is

$$
\begin{aligned}
F(T) & =\frac{4 \mu \epsilon^{2 \nu}}{\Gamma(\nu-1)}(1+\beta)(4 \mu T)^{-\nu-1} e^{-\frac{x^{2}}{4 \mu T}} \\
& \sim T^{-\nu-1}=T^{-\frac{3+\beta}{2}}
\end{aligned}
$$

where $\nu=(1+\beta) / 2$, implying $\alpha=\frac{3+\beta}{2}$. Observe that, in the limit of vanishing potential amplitude, $\lambda=0$, this result reproduces the statistics of a freely-moving random walk, $F(T) \sim T^{-\frac{3}{2}}$, while in the opposite perfectlybalanced limit, $\lambda=\mu$ (i.e. $\beta=1$ ) the result is $F(T) \sim$ $T^{-2}$ in agreement with the expectations for the un-biased branching process. It is noteworthy that -despite the fact that the random walk in a logarithmic potential gives a non-universal avalanche duration exponent- for the undriven DRW case, in which the logarithmic potential derives from a change of variables in Itô calculus, there exists a perfect balance between the coefficients of the equation; they both depend on the noise amplitude and, compensating each other, they generate the universal value $\alpha=2$. However, as said above, in the presence of an external field, $\beta=1-h / \mu$ breaking down the perfect balance between coefficients, non-universal continuouslyvarying avalanche exponents appear (see Figure 3); in particular,

$$
\alpha=2-\frac{h}{2 \mu} .
$$

In any possible discrete/particle model with absorbing states, this change of exponents stems from the fact that -owing to the external driving- avalanches from different initial seeds (each of them spontaneously generated by the external driving field) can merge, which allows their combination to survive longer and be larger, thus leading to smaller effective exponents $\alpha$ and $\tau$ (see Table 1).

Turning back to the general discussion, using the above result together with simple scaling, we can readily derive the associated avalanche size exponent, $\tau$. In order to have a unified notation let us use a generic variable $v(t)$, which can be in particular, $x(t)$ for the RW, or $\rho(t)$ for the DRW. The size of any given avalanche is defined as the area under the curve defined by the random walk, i.e. $S=\int_{0}^{T} v(t) d t$, and we are interested in the distribution of such sizes as a function of $T, P(S \mid T)$. Given that the typical displacement of a random walk in time $t$ scales as $v \sim \sqrt{t}$, for the DRW (for which there is an additional square-root factor) we have $v \sim \sqrt{v} \sqrt{t}$, and thus, $v \sim t$; 

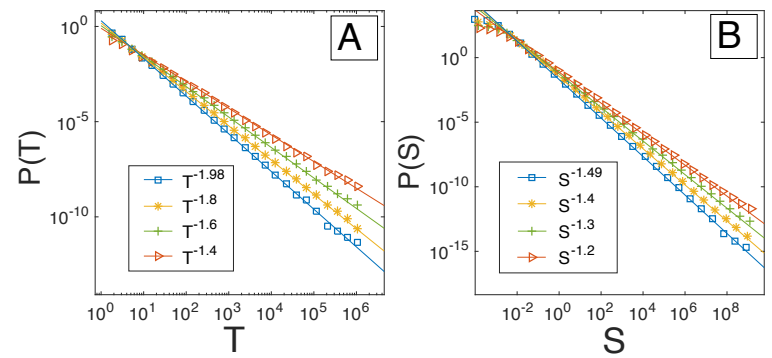

FIG. 3. Size-avalanche and duration-avalanche distributions for the un-driven demographic random walk as described by Eq. 6. , as well as for diverse values of the external driving field (marked with symbols) $h=0.01$ (blue squares), $h=$ 0.1 (yellow stars), $h=0.2$ (green crosses) and $h=0.3$ (red triangles), with reference curves (solid lines) $t^{-2+h /(2 \mu)}$ and $s^{-3 / 2+h /(4 \mu)}$ (as derived in the text), respectively, illustrating the agreement with theoretical predictions.

hence, we can write, in general, $v \sim t^{\phi}$, with $\phi=1 / 2$ and $\phi=1$ for the RW and the DRW (either driven or undriven), respectively.

It is natural to define a new rescaled variable $\tilde{v}(t / T)=$ $v(t) / T^{\phi}$ which describes a random excursion in the interval $[0,1]$. In these terms,

$$
S=\int_{0}^{T} v(t) d t \sim T^{\phi+1} \int_{0}^{1} \tilde{v}(z) d z .
$$

Thus, the average avalanche size, $\langle S\rangle$ obtained averaging over all possible avalanche shapes, $\tilde{v}(z)$, scales also with $T^{\phi+1}$, implying $\gamma=\phi+1$.

Using the previous result, $P(S \mid T)$ can be written as a scaling form $P(S \mid T)=T^{-\gamma} \mathcal{G}\left(S / T^{\gamma}\right)$ where the factor $T^{-\gamma}$ comes from the normalization condition, and the unspecified scaling function $\mathcal{G}$ obeys $\mathcal{G}(z) \geq 0$ for all $z$ and $\int_{0}^{\infty} \mathcal{G}(z) d z=1$. Having computed the conditional probability $P(S \mid T)$, we can explicitly obtain $P(S)$ as

$$
\begin{aligned}
P(S) & =\int_{0}^{\infty} d T P(S \mid T) F(T) \\
& \sim C \int_{0}^{\infty} d T T^{-\gamma} T^{-\alpha} \mathcal{G}\left(S / T^{\gamma}\right) \\
& \sim C S^{-(\gamma+\alpha-1) / \gamma} \int_{0}^{\infty} d u u^{\frac{(\alpha-1)}{\gamma}} \mathcal{G}(u),
\end{aligned}
$$

and, thus, $\tau=(\gamma+\alpha-1) / \gamma$ (which is nothing but the scaling relation Eq.(3)). Plugging the value of $\alpha$ and $\gamma$ derived above one obtains the well-known result $\tau=4 / 3$ for the standard random walk 41] and

$$
\tau=\frac{3}{2}-\frac{h}{4 \mu},
$$

for the DRW, which reduces to the well-known result $\tau=$ $3 / 2$ for the un-driven case. Table 1 contains a summary of the exponents for the different cases.

Results beyond critical exponents have also been obtained in the literature, for example, the average shape of

\begin{tabular}{lccc} 
& Unbiased & Demographic & Driven \\
RW & RW & demographic RW \\
\hline$P(T) \sim T^{-\alpha}$ & $\alpha=3 / 2$ & $\alpha=2$ & $\alpha=2-h / 2 \mu$ \\
\hline$P(S) \sim S^{-\tau}$ & $\tau=4 / 3$ & $\tau=3 / 2$ & $\tau=3 / 2-h / 4 \mu$ \\
\hline$P(S \mid T) \sim T^{-\gamma}$ & $\gamma=3 / 2$ & $\gamma=2$ & $\gamma=2$ \\
\hline
\end{tabular}

TABLE I. Summary of the avalanche exponents for the standard RW, for the demographic RW, and for the driven demographic RW (in the presence of an external field, allowing for the spontaneous generation of activity at a fixed rate $h$ ).

random-walk excursions is a semi-circle for standard unbiased random walkers 21] while it is a parabola for demographic walkers 42]. This can be easily seen by rescaling the walks to $\tilde{v}$ and the times to $t / T$ to collapse curves as described above. In this way $\tilde{v}(t / T)=\mathcal{F}(t / T)$ where $\mathcal{F}(t / T)$ is a scaling function. Given that, $v(t) \sim t^{\gamma-1}$, dividing by $T^{\gamma-1}, \tilde{v}(t / T) \sim(t / T)^{\gamma-1}$, at least for small times, $t<<T$. Considering that a similar relation holds for the reverse time walk starting from $t / T=1$, then the avalanche shape is $\mathcal{F}(t / T)=[(t / T)(1-t / T)]^{\gamma-1}$ which is a semicircle for $\gamma=3 / 2$ (RW) and a parabola for $\gamma=2$ (DRW and driven DRW).

In summary, we have explicitly shown that the meanfield values of avalanche exponents in systems with absorbing states can be computed in a general way by mapping them into a random walk confined by a logarithmic potential, Eq. (8). Of course, this same conclusion could have been reached by arguing in a heuristic way that all of high-dimensional processes involving absorbing states should be effectively described by an un-biased branching process, and then constructing a continuous description of it (i.e. a Fokker-Planck or equivalently a Langevin equation) which would be nothing but Eq. (6).

An interesting corollary is that the exponents do change in the presence of spontaneous creation of activity, even if the rate is arbitrarily small. This result, which stems from the marginality of the associated logarithmic potential could be relevant to understand empirical results; for instance in cortical networks, avalanches of neural activity have been reported to exhibit branching process statistics [43]; still inspection of some of the most careful estimations reveals possible deviations from $\tau=3 / 2$ [44, which could be potentially ascribable to a non-vanishing inherent spontaneous-activation.

We hope that this short paper will help avoiding in the future the frequent confusion we have encountered (mostly in the neuroscience literature) about branching processes and their relation with random walks and also in interpreting empirical results considering the possibility of non-universal continuously-varying exponents. 


\section{Appendix A: Irrelevance of non-linear terms}

For the directed percolation class in the mean-field limit, where spatial heterogeneity is neglected, Eq. (1) reduces to

$$
\dot{\rho}(t)=a \rho-b \rho^{2}+\sqrt{\rho} \eta(t) .
$$

At criticality, i.e. $a=0$, there is still a non-linear (saturation) term $-b \rho^{2}$ which introduces a characteristic maximal activity scale, thus apparently precluding scaleinvariance. The way out of this apparent conundrum is that when studying avalanches in discrete/particle models, activity is created at a single location, and in the continuous limit, this corresponds to vanishing density of activity, $\rho=0$. Thus, one needs to consider a large but finite system size, say $\Omega$ (e.g. one could think of a fully connected network with $\Omega$ nodes), and perform a finitesize scaling analysis. Defining $y$ by $\rho=y / \Omega$ then - up to leading order in $\Omega$ - Eq. A1 reduces to $\dot{y}(\tilde{t})=\sqrt{y} \eta(\tilde{t})$ where $\tilde{t}=\Omega t$. In other words, employing the correct rescaled variables $y$ and $\tilde{t}$ the saturation term is never "seen" by the expanding avalanche, which is compatible with the density being equal to zero, as the avalanche invades an infinitely large system. Observe that in the main text we keep the notation with $\rho$ and $t$, for the sake of simplicity.

Similarly, the voter-model (or compact directed percolation [45] or neutral theory) class - characterized by two symmetric absorbing states- is described, as said above, by the Langevin equation [14]

$$
\left.\dot{\rho}(t)=D \nabla^{2} \rho(\mathbf{r}, t)+\sqrt{\rho(1-\rho}\right) \eta(\mathbf{r}, t),
$$

which, again, ignoring spatial dependencies and rescaling the variables, readily becomes the DRW equation, Eq. 6. The very same reasoning applies also to the other universality classes discussed in the Introduction (i.e. dynamical percolation and the Manna class); also in these cases the corresponding non-linear terms, describing saturation effects vanish upon properly rescaling the system.

On the other hand, beyond the mean-field limit, the non-linearities are essential and control the "renormalized" values of the avalanche exponents (see e.g. [46]), which differ for the various universality classes [22, 24], and avalanches can develop non-symmetric shapes [47].

\section{Appendix B: First-return time distributions}

Following the general result of A. Bray 39 (see also F. Colaiori [40]), here we summarize the computation of avalanche exponents for a random walk in a logarithmic potential. The general Fokker-Plank equation reads 37.

$$
\frac{\partial P(x, t)}{\partial t}=\mu \frac{\partial}{\partial x}\left(\frac{\partial P(x, t)}{\partial x}+\frac{\beta}{x} P(x, t)\right) .
$$

To calculate the probability distribution $F(T)$ of the return times at which a walker starting close to the origin $(P(x, 0)=\delta(x-\epsilon), \epsilon \rightarrow 0)$ first hits back the origin, the absorbing boundary condition $P(0, t)=0$ needs to be imposed. Note that $F(T)$ is minus the probability flux at $0, F(T)=-j(0, t=T)$, with

$$
j(0, t=T)=-\mu\left[\frac{\partial P(x, t)}{\partial x}+\frac{\beta}{x} P(x, t)\right]_{x=0} .
$$

One can try a solution of the Eq.(B1) of the form $P(x, t)=r(x) \exp \left(-\mu k^{2} t\right)$ and note that the resulting equation can be converted into a Bessel Equation with the change of variable $r(x)=x^{\frac{1-\beta}{2}} R(x)$,

$$
x^{2} R^{\prime \prime}(x)+x R^{\prime}(x)+\left(k^{2} x^{2}-\nu^{2}\right) R(x)=0,
$$

where $\nu=(1+\beta) / 2$. The general solution of this last equation is a linear combination of Bessel functions of the first kind of order $\pm \nu$. Putting the pieces back together, employing the orthogonality property of the Bessel functions, and imposing the initial condition, leads to

$$
\begin{aligned}
P(x, t \mid \epsilon, 0) & =\left(\frac{x}{\epsilon}\right)^{1-\nu} \epsilon \int_{0}^{\infty} d k k\left[A J_{\nu}(k \epsilon) J_{\nu}(k x)\right. \\
& \left.+B J_{-\nu}(k \epsilon) J_{-\nu}(k x)\right] e^{-\mu k^{2} t}
\end{aligned}
$$

where $A$ and $B$ are numerical constants. The integral in Eq. B4 gives the modified Bessel function of the first kind $I_{ \pm \nu}$ and, it is easy to compute the flux at the origin in the small $\epsilon$ limit [39, 40, leading to Eq. (10).

\section{ACKNOWLEDGMENTS}

We are grateful to the Spanish-MINECO for financial support (under grant FIS2013-43201-P; FEDER funds). We warmly thank Francesca Colaiori, Jordi Hidalgo, and Paolo Moretti, for very useful comments and suggestions.
[1] T. Liggett, Interacting Particle Systems, Classics in Mathematics (Springer, New York, 2004).
[2] T. E. Harris, The theory of branching processes (Dover, New York, 1989). 
[3] J. Marro and R. Dickman, Nonequilibrium Phase Transition in Lattice Models (Cambridge University Press, Cambridge, 1999).

[4] M. Henkel, H. Hinrichsen, and S. Lübeck, Nonequilibrium Phase Transitions: Absorbing phase transitions, Theoretical and mathematical physics (Springer London, Berlin, 2008).

[5] G. Ódor, Universality in Nonequilibrium Lattice Systems: Theoretical Foundations (World Scientific Publishing, Singapore, 2008).

[6] G. Grinstein and M. A. Muñoz, in Fourth Granada Lectures in Computational Physics, Lecture Notes in Physics, Vol. 493, edited by P. Garrido and J. Marro (1996) p. 223.

[7] K. A. Takeuchi, M. Kuroda, H. Chaté, and M. Sano, Phys. Rev. Lett. 99, 234503 (2007).

[8] H. K. Janssen, Z. Phys. B. 42, 151 (1981).

[9] P. Grassberger, Z. Phys. B. 47, 365 (1982).

[10] J. Binney, N. Dowrick, A. Fisher, and M. Newman, The Theory of Critical Phenomena (Oxford University Press, Oxford, 1993).

[11] Another group of universal behavior is that of systems with noise proportional to the activity (rather that to the square-root of the activity); these encode a different type of processes where the most dominant fluctuations are not demographic, but associated to spatio-temporal variability in the overall parameters [48, 50].

[12] R. Dickman and A. Y. Tretyakov, Phys. Rev. E 52, 3218 (1995).

[13] I. Dornic, H. Chaté, J. Chave, and H. Hinrichsen, Phys. Rev. Lett. 87, 045701 (2001).

[14] O. Al Hammal, H. Chaté, I. Dornic, and M. A. Muñoz, Phys. Rev. Lett. 94, 230601 (2005).

[15] P. Grassberger, Math. Bio. 63, 157 (1983).

[16] H. K. Janssen, Z. Phys. B. 58, 311 (1985).

[17] A. Vespignani, R. Dickman, M. A. Muñoz, and S. Zapperi, Phys. Rev. Lett. 81, 5676 (1998).

[18] J. A. Bonachela and M. A. Muñoz, J. Stat. Mech. 2009, P09009 (2009).

[19] L. P. Kadanoff, S. R. Nagel, L. Wu, and S.-m. Zhou, Phys. Rev. A 39, 6524 (1989).

[20] J. P. Sethna, K. A. Dahmen, and C. R. Myers, Nature 410, 242 (2001).

[21] A. Baldassarri, F. Colaiori, and C. Castellano, Phys. Rev. Lett. 90, 060601 (2003).

[22] M. A. Muñoz, R. Dickman, A. Vespignani, and S. Zapperi, Phys. Rev. E 59, 6175 (1999).

[23] J. Bonachela, Universality in self-organized criticality, Ph.D. thesis, Ph. D. Thesis, University of Granada, Granada, Spain (2008).

[24] S. Lübeck, Int. J. Mod. Phys. B 18, 3977 (2004).

[25] H. N. Huynh and G. Pruessner, Phys. Rev. E 85, 061133 (2012)

[26] H. W. Watson and F. Galton, J. Roy. Anthropol. Inst. 4, 138 (1875).
[27] W. Feller, Ann. Math. , 173 (1951).

[28] P. Hilton and J. Pedersen, Math. Intell. 13, 64 (1991).

[29] N. Dershowitz and C. Rinderknecht, Math. Mag. 88, 187 (2015).

[30] S. Redner, A guide to first-passage processes (Cambridge University Press, Cambridge, 2001).

[31] M. Plischke and B. Bergersen, Equilibrium statistical physics (World Scientific, Singapore, 2006).

[32] S. Zapperi, K. B. Lauritsen, and H. E. Stanley, Phys. Rev. Lett. 75, 4071 (1995).

[33] R. Otter, Ann. Math. Stat. 20, 206 (1949).

[34] M. A. Muñoz, G. Grinstein, and Y. Tu, Phys. Rev. E 56, 5101 (1997).

[35] H. K. Janssen, J. Phys. Cond. Matter 17, S1973 (2005).

[36] N. G. Van Kampen, Stochastic processes in physics and chemistry, Vol. 1 (Elsevier, 1992).

[37] C. Gardiner, Stochastic Methods: A Handbook for the Natural and Social Sciences, Springer Series in Synergetics (Springer, New York, 2009).

[38] An alternative approach to analyze Langevin equations such as Eq. (6) consists in reabsorbing the noise amplitude into the time-scale, leading to a standard random walk with a different "clock" 51. Another interesting possibility is deriving these results from a more general fractional Brownian motion 52.

[39] A. Bray, Phys. Rev. E 62, 103 (2000).

[40] F. Colaiori, Adv. Phys. 57, 287 (2008).

[41] In the case of the standard RW case the scaling function $\mathcal{G}_{\mathcal{R} \mathcal{W}}$ has been exactly derived (see e.g. [53]), but its specific form is not essential for our purposes here.

[42] S. Papanikolaou, F. Bohn, R. L. Sommer, G. Durin, S. Zapperi, and J. P. Sethna, Nat. Phys. 7, 316 (2011).

[43] J. M. Beggs and D. Plenz, J. Neurosci. 23, 11167 (2003).

[44] H. G. Schuster, D. Plenz, and E. Niebur, Criticality in neural systems (John Wiley \& Sons, New Jersey, 2014).

[45] J. Essam, J. Phys. A 22, 4927 (1989).

[46] A. Dobrinevski, P. Le Doussal, and K. J. Wiese, Europhys. Lett. 108, 66002 (2015).

[47] L. Laurson, X. Illa, S. Santucci, K. T. Tallakstad, K. J. Måløy, and M. J. Alava, Nat. Comm. 4 (2013).

[48] G. Grinstein, M. A. Muñoz, and Y. Tu, Phys. Rev. Lett. 76, 4376 (1996).

[49] W. Genovese, M. A. Muñoz, and J. M. Sancho, Phys. Rev. E 57, R2495 (1998).

[50] M. A. Muñoz, F. Colaiori, and C. Castellano, Phys. Rev. E 72, 056102 (2005).

[51] K. J. Rubin, G. Pruessner, and G. A. Pavliotis, J. Phys. A 47, 195001 (2014).

[52] M. Ding and W. Yang, Phys. Rev. E 52, 207 (1995)

[53] S. N. Majumdar and A. Comtet, J. Stat. Phys. 119, 777 (2005). 